Bull.Fac, Agric.,Cairo Univ.,62:173-182 (2011).

\title{
EFFECT OF MYCORRHIZAE, AZOTOBACTER AND ORGANIC MANURE ON THE GROWTH, SEED YIELD AND OIL CONTENT OF Nigella sativa L. PLANTS GROWN UNDER TWO LEVELS OF CHEMICAL FERTILIZERS (NPK)
}

\author{
(Received: 30. 12. 2010)
}

\author{
By \\ E. M. Z. Harb, A.M. Ghallab and S.H.D.Soliman \\ Department of Agricultural Botany, Plant Physiology Division, Faculty of Agriculture, \\ Cairo University, Egypt.
}

\begin{abstract}
Mature black cumin (Nigella sativa L.) seeds were planted in clay loam soil on the $23^{\text {rd }}$ of November, 2005 and 2006 and were harvested on $10^{\text {th }}$ April, 2006 and 2007 seasons. The data of vegetative growth, seed yield and oil seed percentage were determined. The data indicated that the biofertilization (Glomus macrocarpus fungus or Nitrobein bacteria) or organic manure alone or in combination with half or full NPK fertilizer increased plant height $(\mathrm{cm})$, No. of branches and leaves, root length $(\mathrm{cm})$ as well as herb and root dry weight when compared with uninoculated plants (control). Also, the best significant results of herb and root dry weight were found with mycorrhizal fungus and Azotobacter with full NPK fertilizers treatment as compared to the other treatments under study, while, the least values in these respects were found with the control plants. Also, G. macrocarpus fungus + Nitrobein + organic manure with full NPK fertilizer treatment was more effective in increasing the seed yield per plant and feddan than the other treatments under study. The same effect was found with G. macrocarpus fungus alone or combined with Nitrobein treatment when seed total and essential oil content were concerned. Generally, the biofertilization (mycorrhizae or Azotobacter) or organic manure increased the vegetative growth parameters, seed yield, and oil content than the control plants of black cumin.
\end{abstract}

Key words: Azotobacter, chemical fertilizer, mycorrhizae, Niglla sativa, NPK, oil content, organic manure, plant growth, seed yield,.

\section{INTRODUCTION}

Black cumin (Nigella sativa L.) is belonging to family Ranunculaceae,. Black cumin seeds or oil seeds are used by the common people for many medicinal purposes.

Generally, many researchers have been worked on the effect of chemical fertilizers on the growth and seed or oil yield of black cumin plants such as Das et al. (1991) who showed that plant height, no. of branches/plant and fresh or dry weight as well as seed yield of Nigella sativa were increased with $\mathrm{N}$ fertilization at $20-60 \mathrm{~kg} / \mathrm{ha}$ and $\mathrm{P}$ fertilization at $20-40 \mathrm{~kg} / \mathrm{ha}$. El-Deeb et al. (1993) working on Nigella sativa plants showed that application of $\mathrm{N}$ fertilization at any level increased plant height. Singh et al. (1999) found that seed yield of Nigella sativa plants increased as $\mathrm{N}$ and P rates increased. Datta et al. (2001) found that the highest vegetative growth and yield parameters of Nigella sativa plants were obtained with NPK (45-45-45) treatment. Ashraf et al. (2005) reported that the best growth and seed yield of Nigella sativa plants were recorded at 30 and $60 \mathrm{~kg} \mathrm{~N} / \mathrm{ha}$.

Meanwhile, many researches have been worked on the effect of biofertilization and organic manure with or without the addition of chemical fertilization such as Nagarajan et al. (1989) who reported that Azospirillium brasilense and Glomus faciculatum as a combined treatment showed a remarkable increase in shoot and root biomass of mulberry (Morus sp.) in comparison with the uninoculated control plants. Meanwhile, Wange and Patil (1994) applied $100 \mathrm{~kg} \mathrm{~N} / \mathrm{ha}$ alone or inoculating with Azotobacter + Azospirillum mixtures significantly increased the No. of flowers/stable, blub (rhizome) yield and the No. of flower stems produced by Polianthes tuberose plants. Mukhopadhyay and Sem (1997) showed that a significant increases in yield components occurred in black cumin plants with $\mathrm{N}$-fixing bacteria. Also, Gupta et al. (1999) stated that the growth and flower yields of $T$. erecta plants were the highest after treatment with 
Azotobacter + phosphorus solubilizing bacteria in combination with 75 or $100 \% \mathrm{~N}$ application. Also, Salama (2002) studied the response of Balady mandarin trees to organic fertilizers and biofertilizers, and showed that Rhizobacterium produced more enhancing effect on growth cycle duration, growth intensity of different flush, leaf area and leaf dry weight than Nitrobein treatments. Saraf and Tiwari (2004) stated that $75 \% \mathrm{NPK}+\mathrm{FYM}$ at $10 \mathrm{t} / \mathrm{ha}+$ biofertilizers (Azotobacter + phosphate solubilizing bacteria) treatment recorded the highest values of the yield parameters for both the main and ratoon crops of muskdana plants. Ray et al. (2005) reported that the addition of Azotobacter chroococcum and Azospirillum brasilense enhanced the yield of okra plant mere than the uninoculated ones.

The aim of the present work was to study the effect of organic and biofertilization under full or half NPK on growth, yield and chemical components of Nigella sativa.

\section{MATERIALS AND METHODS}

The experimental work was carried out at the Research Centre of the Faculty of Agriculture, Moshtohor, Benha University. The study was conducted through the two successive seasons of 2005-2006 and 2006-2007.

Seeds of black cumin (Nigella sativa L.) were planted on the $23^{\text {rd }}$ of November, 2005 and 2006 at the rate of two plants in each site. The experiment was divided into plots $\left(2 \times 3 \mathrm{~m}^{2}\right)$, each plot contained 6 rows, $50 \mathrm{~cm}$ apart. The distance between the plants was $25 \mathrm{~cm}$. The layout of this experiment was split-split in a complete randomized, design having 16 treatments. They were divided into two groups, each group contained 8 treatments with 6 replicates (6 rows). This experiment was statistically analyzed as a factorial experiment, the main factor was chemical fertilizers (NPK) as two levels, while the branch factor was Mycorrhizae, Azotobacter, organic manure or a combination of them.

The treatments included NPK fertilization according to the recommendation of the Egyptian Ministry of Agriculture $\left(82.5 \mathrm{~kg} \mathrm{~N}, 45 \mathrm{~kg} \mathrm{P}_{2} \mathrm{O}_{5}\right.$ and $36 \mathrm{~kg} \mathrm{~K} \mathrm{~K}_{2} \mathrm{O}$ per feddan) or half of these amounts. The treatments were as follows:

\section{Half NPK}

1- Control (half NPK)

2- Glomus macrocarpus fungus.

3-Nitrobein (N-fixiation bacteria)

\section{Full NPK}

$$
\text { 1-Control (full }
$$$$
\text { (recommended) }
$$

NPK)

2-Glomus macrocarpus fungus.

3-Nitrobein bacteria)
4-Organic
manure (cattle)
5- G.m. + Nitro.
4- Organic manure
6- G.m. + O.m.
(cattle)
7- Nitro + O.m.
5- G.m. + Nitro.
8-G.m.+ Nitro + O.m
6- G.m. + O.m.
7- Nitro + O.m.
8- G.m. + Nitro + O.m.

The seeds of Nigella sativa were planted in clay loam soil. The soil was inoculated before planting with endomycorrhizal fungus (Glomus macrocarpus) according to the method of Menge et al. (1977). While, nitrogen biofertilization treatment was used with Nitrobein bacteria (Azotobacter chroococcum) according to the recommendation of Microbiology Unit, Agric. Research Centre, Egyptian Ministry of Agric. Meanwhile, the organic manure treatment was applied according to the recommendation of the Ministry of Agric. ( $10 \mathrm{~m}^{3} /$ feddan). The nitrogen fertilization was divided into 2 equal portions, the first was applied after one month from seed planting, while the second was applied after 2 months from seed planting as ammonium nitrate $(33 \% \mathrm{~N})$ form. Moreover, calcium superphosphate $\left(15.5 \% \mathrm{P}_{2} \mathrm{O}_{5}\right)$, potassium sulphate $\left(48 \% \mathrm{~K}_{2} \mathrm{O}\right)$, cattle manure, mycorrhizal fungus and Nitrobein bacteria were treated prior to seed planting. However, all the treatments under study were treated with the same agriculture management practies.

Black cumin plants were harvested on 10 April in the first and second seasons (2006 and 2007). The treated plants were subjected to the following measurements:

1- Vegetative growth: plant height $(\mathrm{cm})$, No. of branches and leaves per plant, root length $(\mathrm{cm})$ as well as herb and root dry weight $(\mathrm{g}) / \mathrm{plant}$.

2- The seed yield per plant (g) and per feddan $(\mathrm{kg})$.

3- Total and essential seed oil percentage.

Also, fertilizer dependency ratio (FDR) percentage was calculated on the basis of the ratio between plant fresh weight $(\mathrm{g})$ of fertilizer plants for each mycorrhizal, Nitrobein, organic manure or their combination under full or half NPK and their corresponding control as a formula:

$$
\mathrm{FDR}=\frac{\text { Plant fresh weight of each treatment }}{\text { Controlplant fresh weight (half or full NPK) }} \times 100
$$

Seed oil extracted by using Soxhelet and petroleum ether $\left(40-60^{\circ} \mathrm{C}\right)$ as a solvent according to A.O.A.C. (1985). The total oil percentage of black cumin seeds were calculated. 
Data expressed as percentages were statistically analyzed according to Steel and Torrie (1960). All obtained data were subjected to analysis of variance test and least significant differences among means were calculated according to the Duncan's multiple range tests, Duncan (1955) at $1 \%$ level.

\section{RESULTS AND DISCUSSION \\ 3.1. Growth characters \\ 3.1.1. Plant height and No. of branches}

The data in Table (1) showed that black cumin plants treated with Glomus macrocarpus fungus combined with organic manure (cattle) and mineral fertilizers with full NPK gave the highest values of plant height $(\mathrm{cm})$. The same trend of the highest significant value was observed in No. of branches per plant treated with the interaction treatment (G. macrocarpus + Nitrobein + O.m. with full dose NPK fertilizers) as compared to the other treatments. In contrast, the least values of both plant height and No. of branches were obtained from the control whether full or half dose of NPK fertilizers alone in the two studied seasons.

Also, Mycorrhizal fungus alone or combined with Nitrobein bacteria treatment under any level of mineral fertilizer (half or full) improved plant height $(\mathrm{cm})$ and No. of branches when compared with corresponding plants fertilized with the same level of NPK mineral fertilizer alone.

\subsubsection{Number of leaves per plant and root length (cm)}

The data in Table (2) showed that $G$. macrocarpus fungus + Nitrobein with full dose NPK treatment was more effective in increasing No. of leaves per plant and root length $(\mathrm{cm})$ when compared with the other treatments. While, the least values were obtained with the control whether with full or half dose of NPK.

Meanwhile, when concerning the specific effect of treatments alone, the data revealed that Mycorrhizae or Azotobacter or organic manure induced a significant increase on No. of leaves and root length of Nigella sativa plants than the control plants. In the same time, the biofertilization treatments or organic manure with half NPK fertilizers treatment were reached the same level effect in this respect to the same values of No. of leaves and root length which obsereved with the same biofertilization or organic manure with full dose NPK fertilizers.

3.1.3. Root and herb dry weight of black cumin
The results in Table (3) indicated that the interaction treatment of $G$. macrocarpus fungus + Nitrobein + full dose NPK fertilizers gave the best significant values of herb and root dry weights of Nigella sativa plants. While, the least significant herb and root dry weights were obtained with the control (half NPK treatment).

G. macrocarpus fungus + Nitrobein bacteria treatment was more effective in inducing significant increase in herb and root dry weights more than the other treatments in both seasons.

The specific effect of mycorrhizae or Azotobacter or organic manure gave the best significant herb and root dry weights than the control treatment with NPK fertilizers.

Generaly, the obtained results assured the findings of Gendiah (1987) on sour oranges and Cleopatra mandarin seedlings with mycorrohizal fungi, Rao and Dass (1989) on Zizphus mauritiana cv. Seb and Gola and on root cuttings of pomegranate with Azospirillum and Azotobacter, Nagarajan et al. (1989) on mulberry with Glomus fasiculatum fungus and Azospirillum bacterium, Mousa et al. (2001) and Nataraja et al. (2003) on Nigella sativa plants.

\subsubsection{Fertilizer dependency ratio (FDR)\%}

The results tabulated in Table (4) showed that G. macrocorpus + Nitrobein bacteria with full dose NPK treatment gave the highest significant value of fertilizer dependency ratio percentage of Nigella sativa plants as compared to the other treatments in both seasons. G. macrocorpus fungus + Nitrobein + organic manure + half dose NPK treatment came in the second rank as a significant increase of FDR\% value of black cumin plants. On the contrast, G. macrocarpus fungus + organic manure + half dose NPK treatment gave the least significant value of FDR\% as compared to the other treatments except the control plants as a ratio to the other treatments.

Generally, it might be said that Azotobacter treatment was more effective in increasing the FDR\% values of black cumin plants than mycorrhizal fungus or organic manure. This result might be due to the effect of Nitrobein bacteria on inducing an increase in fresh vegetative growth as compared to other treatments. These results are in agreement with the findings of Gendiah (1987) who found that mycorrhizal dependency percentage values of mycorrhizae treated Cleopatra mandarin and sour oranges were increased in a significant level as compared to untreated seedlings. 
Table (1):Effect of biofertilization (Mycorrhizae or Nitrobein), organic manure or their combinations on plant height and No. of branches of Nigella sativa plants grown under two levels of NPK fertilizers.

\begin{tabular}{|c|c|c|c|c|c|c|c|c|c|c|c|c|}
\hline \multirow{3}{*}{ Treatment } & \multicolumn{6}{|c|}{ Plant height (cm) } & \multicolumn{6}{|c|}{ No. of branches/plant } \\
\hline & \multicolumn{3}{|c|}{$2005-2006$} & \multicolumn{3}{|c|}{ 2006-2007 } & \multicolumn{3}{|c|}{$2005-2006$} & \multicolumn{3}{|c|}{ 2006-2007 } \\
\hline & H.F.* & F.F.** & Mean & H.F.* & F.F.** & Mean & H.F.* & F.F.** & Mean & H.F.* & F.F.** & Mean \\
\hline Control & $44.4 \mathrm{j}$ & $45.6 \mathrm{hij}$ & $45.0 \mathrm{~d}$ & $47.8 \mathrm{~d}$ & $41.2 \mathrm{f}$ & $44.5 \mathrm{f}$ & 3.8 def & 4.6 cde & $4.2 \mathrm{c}$ & $4.0 \mathrm{~d}$ & $4.6 \mathrm{~cd}$ & $4.3 \mathrm{~d}$ \\
\hline Glomus macrocarpus (G.M.) & 49.0 efg & $53.6 \mathrm{~d}$ & $51.3 \mathrm{~b}$ & 58.8 a & $54.8 \mathrm{c}$ & 56.8 a & $6.0 \mathbf{a b}$ & $6.8 \mathrm{a}$ & $6.4 \mathrm{a}$ & $5.0 \mathrm{~cd}$ & $5.6 \mathrm{bcd}$ & $5.3 \mathrm{bcd}$ \\
\hline Nitrobein (Nitro.) & $47.6 \mathrm{fgh}$ & 48.4 efg & $48.0 \mathrm{c}$ & $44.6 \mathrm{e}$ & $55.8 \mathrm{bc}$ & $50.2 \mathrm{~cd}$ & $4.6 \mathrm{bc}$ & 4.4de & $4.5 \mathrm{bc}$ & $6.0 \mathrm{bc}$ & $5.0 \mathrm{~cd}$ & $5.5 \mathrm{bc}$ \\
\hline Organic manure (O.M.) & 50.0 e & $56.2 \mathrm{c}$ & $53.1 \mathrm{~b}$ & $43.6 \mathrm{e}$ & 59.4 a & $51.5 \mathrm{bcd}$ & $5.6 \mathrm{bc}$ & 3.8 def & $4.7 \mathrm{bc}$ & $6.8 \mathrm{ab}$ & $5.0 \mathrm{~cd}$ & $5.9 \mathrm{~b}$ \\
\hline G.M. + Nitro. & $53.6 \mathrm{~d}$ & $60.0 \mathrm{~b}$ & $56.8 \mathrm{a}$ & $45.4 \mathrm{e}$ & 56.2 bc & 50.8 bcd & $4.8 \mathrm{~cd}$ & $4.4 \mathrm{de}$ & $4.6 \mathrm{bc}$ & $4.2 \mathrm{~cd}$ & $6.0 \mathrm{bc}$ & $5.1 \mathrm{bcd}$ \\
\hline G.M. + O.M. & $50.2 \mathrm{e}$ & $63.0 \mathrm{a}$ & 56.6 a & $48.0 \mathrm{~d}$ & $57.8 \mathbf{a b}$ & $52.9 \mathrm{~b}$ & 4.6 cde & $3.2 \mathrm{f}$ & $3.9 \mathrm{c}$ & $5.0 \mathrm{~cd}$ & $4.0 \mathrm{~d}$ & $4.5 \mathrm{~cd}$ \\
\hline Nitro. + O.M. & 47.0 ghi & $58.6 \mathrm{~b}$ & $52.8 \mathrm{~b}$ & $44.8 \mathrm{e}$ & $55.0 \mathrm{c}$ & $49.9 \mathrm{~d}$ & $3.6 \mathrm{ef}$ & 3.8 def & $3.7 \mathrm{c}$ & $5.0 \mathrm{~cd}$ & $4.0 \mathrm{~d}$ & $4.5 \mathrm{~cd}$ \\
\hline G.M. + Nitro. + O.M. & 45.0 ij & 49.6 ef & $47.3 \mathrm{c}$ & $43.4 \mathrm{e}$ & 58.8 a & $51.1 \mathrm{bcd}$ & $4.8 \mathrm{~cd}$ & $6.0 \mathrm{ab}$ & $5.4 \mathrm{ab}$ & $7.0 \mathrm{ab}$ & $8.0 \mathrm{a}$ & $7.5 \mathrm{a}$ \\
\hline \multicolumn{13}{|c|}{ Specific effect } \\
\hline NPK (control) & $44.4 \mathrm{c}$ & $45.6 \mathrm{~b}$ & $45.0 \mathrm{~b}$ & $47.8 \mathrm{a}$ & $41.2 \mathrm{~b}$ & $44.5 \mathrm{~b}$ & $3.8 \mathrm{~b}$ & $4.6 \mathrm{a}$ & $4.2 \mathrm{~b}$ & $4.0 \mathrm{~b}$ & $4.6 \mathrm{~b}$ & $4.3 \mathrm{~b}$ \\
\hline Mycorrhizae & $49.5 \mathrm{~b}$ & $56.6 \mathrm{a}$ & 53.1 a & $48.9 \mathrm{a}$ & 56.9 a & 52.9 a & $6.1 \mathrm{a}$ & $5.1 \mathrm{a}$ & $5.6 \mathrm{a}$ & $5.3 \mathrm{a}$ & $5.9 \mathrm{a}$ & $5.6 \mathrm{a}$ \\
\hline Azotobacter & $58.3 \mathrm{a}$ & $54.2 \mathrm{a}$ & $51.3 \mathrm{a}$ & $44.6 \mathrm{~b}$ & $56.5 \mathrm{a}$ & 50.6 a & $4.5 \mathrm{~b}$ & $4.7 \mathrm{a}$ & $4.6 \mathrm{ab}$ & $5.6 \mathrm{a}$ & $5.8 \mathrm{a}$ & $5.7 \mathrm{a}$ \\
\hline Organic manure & $48.1 \mathrm{~b}$ & 56.9 a & $52.5 \mathrm{a}$ & $45.0 \mathrm{~b}$ & 57.8 a & 51.4 a & $4.7 \mathrm{~b}$ & $4.2 \mathrm{a}$ & $4.5 \mathrm{~b}$ & $6.0 \mathrm{a}$ & $5.3 \mathrm{ab}$ & $5.7 \mathrm{a}$ \\
\hline
\end{tabular}

* H.F.: Half dose NPK fertilizers.

** F.F. Full dose NPK fertilizers.

Means followed by the same letter, within each column, are not significantly different for each other at $1 \%$ level. 
Table (2): Effect of biofertilization (Mycorrhizae or Nitrobein), organic manure or their combinations on No. of leaves and root length (cm) of Nigella sativa plants grown under two levels of NPK fertilizers.

\begin{tabular}{|c|c|c|c|c|c|c|c|c|c|c|c|c|}
\hline \multirow{3}{*}{ Treatment } & \multicolumn{6}{|c|}{ No. of leaves/plant } & \multicolumn{6}{|c|}{ Root length (cm) } \\
\hline & \multicolumn{3}{|c|}{$2005-2006$} & \multicolumn{3}{|c|}{ 2006-2007 } & \multicolumn{3}{|c|}{$2005-2006$} & \multicolumn{3}{|c|}{ 2006-2007 } \\
\hline & H.F.* & F.F.** & Mean & H.F.* & F.F.** & Mean & H.F.* & F.F.** & Mean & H.F.* & F.F.** & Mean \\
\hline Control & 14.0 ef & $14.8 \mathrm{e}$ & $14.4 \mathrm{c}$ & $14.2 \mathrm{hi}$ & $15.5 \mathrm{gh}$ & $14.9 \mathrm{~d}$ & 11.3 cde & $9.4 \mathrm{ef}$ & $10.4 \mathrm{~b}$ & 10.0 ef & $7.2 \mathrm{~g}$ & $8.6 \mathrm{c}$ \\
\hline Glomus macrocarpus (G.M.) & $15.0 \mathrm{e}$ & $18.4 \mathrm{~d}$ & $16.7 \mathrm{bc}$ & $16.0 \mathrm{fgh}$ & 18.0 def & $17.0 \mathrm{~cd}$ & 10.0 def & $13.8 \mathrm{ab}$ & $11.9 \mathrm{a}$ & 10.2 ef & 11.4 cde & $10.8 \mathrm{~b}$ \\
\hline Nitrobein (Nitro.) & 14.4 ef & 14.4 ef & $14.4 \mathrm{c}$ & $13.0 \mathrm{i}$ & $20.0 \mathrm{bcd}$ & $16.5 \mathrm{~d}$ & $9.6 \mathrm{ef}$ & $12.6 \mathrm{bc}$ & $11.1 \mathrm{ab}$ & 10.0 ef & $11.2 \mathrm{de}$ & $10.6 \mathrm{~b}$ \\
\hline Organic manure (O.M.) & 14.4 ef & $21.6 \mathrm{~b}$ & $18.0 \mathrm{ab}$ & 17.2 efg & 20.2 bcd & 18.7 bc & 11.0 cde & $12.0 \mathrm{bcd}$ & $11.5 \mathrm{ab}$ & 9.8 ef & $12.0 \mathrm{bcd}$ & $10.9 \mathrm{~b}$ \\
\hline G.M. + Nitro. & $12.6 \mathrm{fg}$ & 24.6 a & $18.6 \mathrm{ab}$ & 19.0 cde & 23.8 a & $21.4 \mathrm{a}$ & 11.0 cde & $13.4 \mathrm{ab}$ & $12.2 \mathrm{a}$ & $10.8 \mathrm{de}$ & $13.8 \mathrm{a}$ & $12.3 \mathrm{ab}$ \\
\hline G.M. + O.M. & $11.6 \mathrm{~g}$ & $19.4 \mathrm{~cd}$ & $15.5 \mathrm{c}$ & 18.8 cde & 18.8 cde & 18.8 bc & $8.4 \mathrm{f}$ & $15.4 \mathrm{a}$ & 11.9 a & 11.0 de & $13.3 \mathrm{ab}$ & $12.2 \mathrm{ab}$ \\
\hline Nitro. + O.M. & $13.2 \mathrm{efg}$ & $23.0 \mathrm{ab}$ & $18.1 \mathrm{ab}$ & $18.3 \mathrm{de}$ & $20.8 \mathrm{bc}$ & $19.6 \mathrm{ab}$ & 9.0 ef & $12.8 \mathrm{bc}$ & 10.9 ab & 9.0f & 12.2 abcd & $10.6 \mathrm{~b}$ \\
\hline G.M. + Nitro. + O.M. & $17.6 \mathrm{~d}$ & $21.2 \mathrm{bc}$ & $19.4 \mathrm{a}$ & 18.0 def & $22.0 \mathrm{ab}$ & $20.0 \mathrm{ab}$ & $12.0 \mathrm{bcd}$ & 11.0 cde & $11.5 \mathrm{ab}$ & $12.0 \mathrm{bcd}$ & 13.0 abc & $12.5 \mathrm{a}$ \\
\hline \multicolumn{13}{|c|}{ Specific effect } \\
\hline NPK (control) & $14.0 \mathrm{a}$ & $14.8 \mathrm{~b}$ & $14.4 \mathrm{~b}$ & $14.2 \mathrm{~b}$ & $15.5 \mathrm{~b}$ & $14.9 \mathrm{~b}$ & $11.3 \mathrm{a}$ & $9.4 \mathrm{~b}$ & $10.4 \mathrm{~b}$ & $10.0 \mathrm{a}$ & $7.2 \mathrm{~b}$ & $8.6 \mathrm{~b}$ \\
\hline Mycorrhizae & $14.2 \mathrm{a}$ & 20.9 a & $17.6 \mathrm{a}$ & $18.0 \mathrm{a}$ & $19.8 \mathrm{a}$ & 18.9 a & $10.4 \mathrm{a}$ & $13.4 \mathrm{a}$ & $11.9 \mathrm{a}$ & $11.0 \mathrm{a}$ & $12.4 \mathrm{a}$ & $11.7 \mathrm{a}$ \\
\hline Azotobacter & $14.5 \mathrm{a}$ & $20.8 \mathrm{a}$ & $17.7 \mathrm{a}$ & $17.1 \mathrm{a}$ & $20.8 \mathrm{a}$ & $19.0 \mathrm{a}$ & $10.4 \mathrm{a}$ & $12.5 \mathrm{a}$ & $11.5 \mathrm{ab}$ & $10.5 \mathrm{a}$ & $12.1 \mathrm{a}$ & $11.3 \mathrm{a}$ \\
\hline Organic manure & $14.2 \mathrm{a}$ & $21.3 \mathrm{a}$ & $17.8 \mathrm{a}$ & 18.1 a & $21.4 \mathrm{a}$ & $19.8 \mathrm{a}$ & 10.1 a & $12.8 \mathrm{a}$ & $11.5 \mathrm{ab}$ & $10.5 \mathrm{a}$ & $13.1 \mathrm{a}$ & $11.8 \mathrm{a}$ \\
\hline
\end{tabular}

* H.F.: Half dose NPK fertilizers.

** F.F.: Full dose NPK fertilizers.

Means followed by the same letter, within each column, are not significantly different for each other at $1 \%$ level. 
Table (3): Effect of biofertilization (Mycorrhizae or Nitrobein), organic manure or their combinations on dry weight of herb and root dry weight (g) of Nigella sativa plants grown under two levels of NPK fertilizers.

\begin{tabular}{|c|c|c|c|c|c|c|c|c|c|c|c|c|}
\hline \multirow{3}{*}{ Treatment } & \multicolumn{6}{|c|}{ Dry weight of herb (g) } & \multicolumn{6}{|c|}{ Root dry weight (g) } \\
\hline & \multicolumn{3}{|c|}{ 2005-2006 } & \multicolumn{3}{|c|}{ 2006-2007 } & \multicolumn{3}{|c|}{ 2005-2006 } & \multicolumn{3}{|c|}{ 2006-2007 } \\
\hline & H.F.* & F.F.** & Mean & H.F.* & F.F.** & Mean & H.F.* & F.F.** & Mean & H.F.* & F.F.** & Mean \\
\hline Control & $2.43 \mathrm{~g}$ & $3.54 \mathrm{f}$ & $3.00 \mathrm{e}$ & $1.61 \mathrm{~g}$ & $2.34 \mathrm{f}$ & $1.98 \mathrm{c}$ & 0.13 e & $0.21 \mathrm{de}$ & $0.17 \mathrm{c}$ & $0.14 \mathrm{~g}$ & 0.33 de & $0.24 \mathrm{~b}$ \\
\hline Glomus macrocarpus (G.M.) & $2.74 \mathrm{~g}$ & $5.09 \mathrm{~cd}$ & $3.92 \mathrm{~cd}$ & $3.30 \mathrm{e}$ & $4.41 \mathrm{~d}$ & $3.86 \mathrm{~b}$ & 0.14 e & $0.41 \mathrm{~b}$ & $0.28 \mathrm{ab}$ & $0.20 \mathrm{fg}$ & $0.39 \mathrm{~cd}$ & $0.30 \mathrm{ab}$ \\
\hline Nitrobein (Nitro.) & $2.88 \mathrm{~g}$ & $5.89 \mathrm{~b}$ & $4.39 \mathrm{bc}$ & $3.31 \mathrm{e}$ & $6.10 \mathrm{~b}$ & $4.71 \mathrm{a}$ & 0.19 de & $0.44 \mathrm{ab}$ & $0.32 \mathrm{ab}$ & $0.21 \mathrm{fg}$ & 0.44 bc & $0.33 \mathrm{a}$ \\
\hline Organic manure (O.M.) & $4.45 \mathrm{de}$ & $6.43 \mathrm{a}$ & $5.44 \mathrm{a}$ & $2.54 \mathrm{f}$ & $6.17 \mathrm{~b}$ & $4.36 \mathrm{ab}$ & 0.23 cde & $0.40 \mathrm{~b}$ & $0.32 \mathrm{ab}$ & $0.14 \mathrm{~g}$ & $0.50 \mathrm{ab}$ & $0.32 \mathrm{ab}$ \\
\hline G.M. + Nitro. & $3.60 \mathrm{f}$ & $7.21 \mathrm{a}$ & $5.41 \mathrm{a}$ & $2.52 \mathrm{f}$ & $6.91 \mathrm{a}$ & $4.72 \mathrm{a}$ & 0.19 de & 0.52 a & $0.36 \mathrm{a}$ & $0.16 \mathrm{~g}$ & $0.53 \mathrm{a}$ & $0.35 \mathrm{a}$ \\
\hline G.M. + O.M. & $2.25 \mathrm{~g}$ & $4.97 \mathrm{~d}$ & $3.61 \mathrm{de}$ & $4.50 \mathrm{~cd}$ & $5.01 \mathrm{~cd}$ & $4.76 \mathrm{a}$ & $0.21 \mathrm{de}$ & 0.26 cde & $0.24 \mathrm{bc}$ & $0.36 \mathrm{~cd}$ & $0.37 \mathrm{~cd}$ & $0.37 \mathrm{a}$ \\
\hline Nitro. + O.M. & $2.88 \mathrm{~g}$ & $5.92 \mathrm{~b}$ & $4.40 \mathrm{bc}$ & $3.24 \mathrm{e}$ & $5.17 \mathrm{c}$ & $4.21 \mathrm{ab}$ & 0.16 de & $0.39 \mathrm{~b}$ & $0.28 \mathrm{ab}$ & $0.22 \mathrm{fg}$ & $0.51 \mathrm{ab}$ & $0.37 \mathrm{a}$ \\
\hline G.M. + Nitro. + O.M. & $4.11 \mathrm{ef}$ & $5.24 \mathrm{~cd}$ & $4.68 \mathrm{~b}$ & $3.16 \mathrm{e}$ & $4.61 \mathrm{~cd}$ & $3.89 \mathrm{~b}$ & $0.28 \mathrm{~cd}$ & 0.34 bc & $0.31 \mathrm{ab}$ & 0.26 ef & $0.34 \mathrm{de}$ & $0.30 \mathrm{ab}$ \\
\hline \multicolumn{13}{|c|}{ Specific effect } \\
\hline NPK (control) & $2.43 \mathrm{~b}$ & $3.54 \mathrm{~b}$ & $3.00 \mathrm{~b}$ & $1.61 \mathrm{~b}$ & $2.34 \mathrm{~b}$ & $1.98 \mathrm{~b}$ & $0.13 \mathrm{~b}$ & $0.21 \mathrm{c}$ & 0.17 b & 0.14 b & 0.33 b & $0.24 \mathrm{~b}$ \\
\hline Mycorrhizae & $3.18 \mathrm{a}$ & $5.63 \mathrm{a}$ & $4.41 \mathrm{a}$ & 3.37 a & $5.24 \mathrm{a}$ & $4.31 \mathrm{a}$ & 0.21 a & 0.38 ab & 0.30 a & $0.25 \mathrm{a}$ & 0.41 a & $0.33 \mathrm{a}$ \\
\hline Azotobacter & $3.37 \mathbf{a}$ & 6.07 a & $4.72 \mathrm{a}$ & $3.06 \mathrm{a}$ & $5.70 \mathrm{a}$ & $4.38 \mathrm{a}$ & $0.21 \mathrm{a}$ & $0.42 \mathrm{a}$ & 0.32 a & $0.21 \mathrm{a}$ & $0.46 \mathrm{a}$ & $0.34 \mathrm{a}$ \\
\hline Organic manure & $3.42 \mathrm{a}$ & $5.64 \mathrm{a}$ & $4.53 \mathrm{a}$ & $3.36 \mathrm{a}$ & $5.24 \mathrm{a}$ & $4.30 \mathrm{a}$ & $0.22 \mathrm{a}$ & $0.35 \mathrm{~b}$ & $0.29 \mathrm{a}$ & $0.25 \mathrm{a}$ & $0.43 \mathrm{a}$ & $0.34 \mathrm{a}$ \\
\hline
\end{tabular}

* H.F.: Half dose NPK fertilizers.

** F.F.: Full dose NPK fertilizers.

Means followed by the same letter, within each column, are not significantly different for each other at $1 \%$ level. 
Table (4): Effect of biofertilization (Mycorrhizae or Nitrobein), organic manure or their combinations on fertilizer dependency ratio (FDR) \% of Nigella sativa plants grown under two levels of NPK fertilizers.

\begin{tabular}{|c|c|c|c|c|c|c|}
\hline \multirow{3}{*}{ Treatment } & \multicolumn{6}{|c|}{ Fertilizer dependcy ration \% (FDR) } \\
\hline & \multicolumn{3}{|c|}{ 2005-2006 } & \multicolumn{3}{|c|}{ 2006-2007 } \\
\hline & H.F.* & F.F.** & Mean & H.F.* & F.F.** & Mean \\
\hline Control & $0.00 \mathrm{j}$ & $0.00 \mathrm{j}$ & $0.00 \mathrm{f}$ & $0.00 \mathrm{j}$ & $0.00 \mathrm{j}$ & $0.00 \mathrm{e}$ \\
\hline $\begin{array}{l}\text { Glomus } \\
\text { macrocarpus (G.M.) }\end{array}$ & $39.27 \mathrm{hi}$ & $77.46 \mathrm{~g}$ & $58.37 \mathrm{e}$ & $95.60 \mathrm{f}$ & $72.98 \mathrm{~g}$ & $84.29 \mathrm{c}$ \\
\hline Nitrobein (Nitro.) & $54.62 \mathrm{~h}$ & $115.45 \mathrm{bc}$ & $85.04 \mathrm{c}$ & $89.36 \mathrm{f}$ & $111.36 \mathrm{e}$ & $100.36 \mathrm{~b}$ \\
\hline $\begin{array}{l}\text { Organic manure } \\
\text { (O.M.) }\end{array}$ & $118.86 \mathrm{~b}$ & 89.93 ef & $104.40 \mathrm{~b}$ & $45.11 \mathrm{~h}$ & $89.77 \mathrm{f}$ & $67.44 \mathrm{~d}$ \\
\hline G.M. + Nitro. & $106.63 \mathrm{~cd}$ & $182.46 \mathrm{a}$ & $144.55 \mathrm{a}$ & $123.36 \mathrm{~cd}$ & $186.81 \mathrm{a}$ & $155.09 \mathrm{a}$ \\
\hline G.M. + O.M. & $47.98 \mathrm{hi}$ & $90.73 \mathrm{ef}$ & $69.36 \mathrm{~d}$ & $17.59 \mathrm{i}$ & $138.25 \mathrm{~b}$ & $77.92 \mathrm{c}$ \\
\hline Nitro. + O.M. & $100.39 \mathrm{de}$ & $86.84 \mathrm{fg}$ & $93.62 \mathrm{c}$ & $129.36 \mathrm{bc}$ & $179.29 \mathrm{a}$ & $154.33 \mathrm{a}$ \\
\hline G.M.+ Nitro.+ O.M. & $125.88 \mathrm{~b}$ & $104.81 \mathrm{~cd}$ & $115.35 \mathrm{~b}$ & $119.29 \mathrm{de}$ & $127.27 \mathrm{~cd}$ & $123.28 \mathrm{~b}$ \\
\hline \multicolumn{7}{|c|}{ Specific effect } \\
\hline NPK (control) & $0.00 \mathrm{c}$ & $0.00 \mathrm{c}$ & $0.00 \mathrm{c}$ & $0.00 \mathrm{~d}$ & $0.00 \mathrm{c}$ & $0.00 \mathrm{c}$ \\
\hline Mycorrhizae & $79.94 \mathrm{~b}$ & $113.87 \mathrm{a}$ & $96.91 \mathrm{~b}$ & $88.96 \mathrm{~b}$ & $131.33 \mathrm{~b}$ & $110.15 \mathrm{~b}$ \\
\hline Azotobacter & $96.88 \mathrm{a}$ & $122.39 \mathrm{a}$ & $109.64 \mathrm{a}$ & $115.34 \mathrm{a}$ & $151.18 \mathrm{a}$ & $133.26 \mathrm{a}$ \\
\hline Organic manure & $98.28 \mathrm{a}$ & $93.08 \mathrm{~b}$ & $95.68 \mathrm{~b}$ & $77.84 \mathrm{c}$ & $133.65 \mathrm{~b}$ & $105.75 \mathrm{~b}$ \\
\hline
\end{tabular}

* H.F.: Half dose NPK fertilizers. ** F.F.: Full dose NPK fertilizers.

Means followed by the same letter, within each column, are not significantly different fro each other at $1 \%$ level.

\subsection{Seed yield/feddan}

The results in Table (5) showed that $G$. macrocapus fungus with a half dose NPK fertilizers or G. macrocarpus fungus + Nitrobein bacteria + organic manure with full NPK gave a higher significant seed yield per plant or feddan as compared to the other treatments in both seasons. On the contrast, the control plants fertilized with half NPK gave the least yield per plant or feddan of black cumin plants.

These results agree with the findings of Das et al. (1991) and Singh et al. (1999) on the effects of chemical fertilizers $\mathrm{N}$ and $\mathrm{P}$ on Nigella sativa plant yield, Srivastava et al. (2002) on organic manure fertilizers and mycorrhizal fungi with citrus trees. Also, Saraf and Tiwari (2004) stated that $75 \% \mathrm{NPK}+\mathrm{FYM}$ at $10 \mathrm{t} / \mathrm{ha}+$ biofertilizers (Azotobacter + phosphate solubilizing bacteria) treatment recorded the highest values for No. of fruits/plant, fruit length, fruit diameter, fruit weight, seed number per fruit, seed weight/fruit and seed yield for both the main and ratoom crops of muskdana plants.

\subsection{Total seed oil percentage}

The results in Table (6) showed that $G$. macrocarpus fungus with half NPK treatment or mycorrhizal fungus + Nitrobein with half NPK gave the highest significant values of seed oil percentage as compared to the other treatments.
While, the least value of seed total oil percentage was observed with the control plants fertilized with half NPK.

Moreover, the biofertilization with endomycorrhizae or Nitrobein (Azotobacter) as well as organic fertilization with cattle manure for Nigella sativa plants induced highly significant values of seed oil percentage more than uninoculated plants fertilized only with chemical NPK. Also, mycorrhizal fungus treated plants and fertilized with half NPK produced higher seed oil percentage comparing with the analogous ones (mycorrhizal plants) with full NPK treatments.

The obtained results on black cumin plants are in agreement with the findings of Akath Singh and Singh (2004) who found that the treatment of three-fourth dose $\mathrm{N}+$ full dose $\mathrm{P}+20 \mathrm{~g}$ Azotobacter/tree of olive trees gave the highest increase in oil content of fruits. Also, Khalid et al. (2005) reported that celery plants treated with S and S-oxidizing bacteria (Thiobacillus thiooxidans) increased total fixed and essential oil contents than the control plants.

Generally, the combination of organic and biofertilizers under half dose of NPK resulted in beneficial effects on increasing seed yield. This might allow the reduction of chemical fertilization saving money and biohazard environment pollution. 
Table (5): Effect of biofertilization (Mycorrhizae or Nitrobein), organic manure or their combinations on the yield of plant (g) and yield/feddan (kg) of seed Nigella sativa plants grown under two levels of NPK fertilizers.

\begin{tabular}{|c|c|c|c|c|c|c|c|c|c|c|c|c|}
\hline \multirow{3}{*}{ Treatments } & \multicolumn{6}{|c|}{ The seed yield of plant (g) } & \multicolumn{6}{|c|}{ The seed yield/feddan $(\mathrm{kg})$} \\
\hline & \multicolumn{3}{|c|}{$2005-2006$} & \multicolumn{3}{|c|}{ 2006-2007 } & \multicolumn{3}{|c|}{ 2005-2006 } & \multicolumn{3}{|c|}{ 2006-2007 } \\
\hline & H.F.* & F.F.** & Mean & H.F.* & F.F.** & Mean & H.F.* & F.F.** & Mean & H.F.* & F.F.** & Mean \\
\hline Control & $2.916 \mathrm{~g}$ & $3.625 \mathrm{f}$ & $3.271 \mathrm{f}$ & $3.010 \mathrm{~h}$ & $3.920 \mathrm{~g}$ & $3.465 \mathrm{e}$ & $168.0 \mathrm{j}$ & $208.8 \mathrm{i}$ & $188.4 \mathrm{e}$ & $173.4 \mathrm{i}$ & $225.8 \mathrm{~h}$ & $199.6 \mathrm{e}$ \\
\hline Glomus macrocarpus (G.M.) & $5.875 \mathrm{ab}$ & 5.416 bc & $5.646 \mathrm{ab}$ & $6.800 \mathrm{a}$ & $5.810 \mathrm{~cd}$ & $6.305 \mathrm{a}$ & 338.4 bc & 312.0 de & $325.2 \mathrm{a}$ & $391.7 \mathrm{a}$ & 334.7 de & $363.2 \mathrm{a}$ \\
\hline Nitrobein (Nitro.) & $4.583 \mathrm{de}$ & 4.083 ef & $4.333 \mathrm{e}$ & $5.020 \mathrm{e}$ & $4.200 \mathrm{fg}$ & $4.660 \mathrm{~cd}$ & $264.0 \mathrm{~g}$ & $235.2 \mathrm{~h}$ & $249.6 \mathrm{~d}$ & $289.2 \mathrm{f}$ & $247.7 \mathrm{~g}$ & $268.5 \mathrm{c}$ \\
\hline Organic manure (O.M.) & $3.750 \mathrm{f}$ & $4.500 \mathrm{e}$ & $4.125 \mathrm{e}$ & $4.200 \mathrm{~g}$ & $4.200 \mathrm{~g}$ & $4.200 \mathrm{~d}$ & $216.0 \mathrm{hi}$ & $259.2 \mathrm{~g}$ & $237.6 \mathrm{~d}$ & $241.9 \mathrm{gh}$ & $241.9 \mathrm{gh}$ & $241.9 d$ \\
\hline G.M. + Nitro. & 5.000 cde & $5.583 \mathrm{~b}$ & $5.292 \mathrm{bc}$ & $5.600 \mathrm{~d}$ & $5.700 \mathrm{~cd}$ & $5.650 \mathrm{~b}$ & $288.0 \mathrm{f}$ & $321.6 \mathrm{~cd}$ & $304.8 \mathrm{~b}$ & $322.6 \mathrm{e}$ & $328.3 \mathrm{e}$ & $325.5 \mathrm{~b}$ \\
\hline G.M. + O.M. & $3.833 \mathrm{f}$ & $5.958 \mathrm{ab}$ & $4.896 \mathrm{~cd}$ & 4.800 ef & $6.100 \mathrm{bcd}$ & $5.450 \mathrm{~b}$ & $220.0 \mathrm{hi}$ & $343.2 \mathrm{~b}$ & $282.0 \mathrm{c}$ & $276.5 \mathrm{f}$ & $351.4 \mathrm{~cd}$ & $313.9 \mathrm{~b}$ \\
\hline Nitro. + O.M. & $5.041 \mathrm{~cd}$ & $3.916 \mathrm{f}$ & 4.479 de & $5.600 \mathrm{~d}$ & $4.010 \mathrm{~g}$ & $4.805 \mathrm{c}$ & 290.4 ef & $225.6 \mathrm{hi}$ & $258.0 \mathrm{~d}$ & $322.6 \mathrm{e}$ & $231.0 \mathrm{gh}$ & $276.8 \mathrm{c}$ \\
\hline G.M. + Nitro. + O.M. & $5.333 \mathrm{c}$ & $6.333 \mathrm{a}$ & $5.833 \mathrm{a}$ & $\begin{array}{l}6.200 \\
\text { bc }\end{array}$ & $6.500 \mathrm{ab}$ & $6.350 \mathrm{a}$ & 307.2 def & $364.8 \mathrm{a}$ & 336.0 a & 357.1 bc & $374.4 \mathrm{ab}$ & $365.8 \mathrm{a}$ \\
\hline \multicolumn{13}{|c|}{ Specific effect } \\
\hline NPK (control) & $2.916 \mathrm{c}$ & $3.625 \mathrm{c}$ & $3.271 \mathrm{c}$ & $3.010 \mathrm{~b}$ & $3.920 \mathrm{c}$ & $3.465 \mathrm{c}$ & $168.0 \mathrm{c}$ & $208.8 \mathrm{c}$ & $188.4 \mathrm{c}$ & $173.4 \mathrm{c}$ & $225.8 \mathrm{c}$ & $199.6 \mathrm{c}$ \\
\hline Mycorrhizae & $5.010 \mathrm{a}$ & $5.823 \mathrm{a}$ & $5.417 \mathrm{a}$ & $5.850 \mathrm{a}$ & $6.028 \mathrm{a}$ & 5.939 a & 288.6 a & $335.4 \mathrm{a}$ & $312.0 \mathrm{a}$ & $337.0 \mathrm{a}$ & $347.2 \mathrm{a}$ & 342.1 a \\
\hline Azotobacter & $4.989 \mathrm{ab}$ & $4.979 \mathrm{~b}$ & $4.984 \mathrm{ab}$ & $5.605 \mathrm{a}$ & $5.103 \mathrm{~b}$ & $5.354 \mathrm{~b}$ & $287.4 \mathrm{a}$ & $286.8 \mathrm{~b}$ & $287.1 \mathrm{~b}$ & $322.9 \mathrm{a}$ & $295.4 \mathrm{~b}$ & $309.2 \mathrm{~b}$ \\
\hline Organic manure & $4.489 \mathrm{~b}$ & $5.177 \mathrm{ab}$ & $4.833 \mathrm{~b}$ & $5.200 \mathrm{a}$ & $5.203 \mathrm{~b}$ & $5.202 \mathrm{~b}$ & $258.6 \mathrm{~b}$ & $298.2 \mathrm{~b}$ & $278.4 \mathrm{~b}$ & $299.5 \mathrm{~b}$ & $299.7 \mathrm{~b}$ & $299.6 \mathrm{~b}$ \\
\hline
\end{tabular}

* H.F.: Half dose NPK fertilizers.

** F.F.: Full dose NPK fertilizers.

Means followed by the same letter, within each column, are not significantly different for each other at $1 \%$ level. 
Table (6): Effect of biofertilization (Mycorrhizae and Nitrobein) and organic manure on seed oil (\%) of Nigella sativa plants grown under two levels of NPK fertilizers.

\begin{tabular}{|c|c|c|c|c|c|c|}
\hline \multirow{3}{*}{ Treatment } & \multicolumn{6}{|c|}{ Total seed oil (\%) } \\
\hline & \multicolumn{3}{|c|}{ 2005-2006 } & \multicolumn{3}{|c|}{ 2006-2007 } \\
\hline & H.F.* & F.F.** & Mean & H.F.* & F.F.** & Mean \\
\hline Control & $24.98 \mathrm{~g}$ & $25.67 \mathrm{~g}$ & $25.33 \mathrm{e}$ & $25.18 \mathrm{j}$ & $27.18 \mathrm{ij}$ & $26.18 \mathrm{e}$ \\
\hline macrocarpus & $38.21 \mathrm{a}$ & $30.85 \mathrm{cde}$ & $34.53 \mathrm{ab}$ & $37.71 \mathrm{ab}$ & $32.35 \mathrm{fg}$ & $35.03 \mathrm{ab}$ \\
\hline Nitrobein (Nitro.) & $31.40 \mathrm{bcd}$ & 30.34 cde & $30.87 \mathrm{c}$ & $32.15 \mathrm{fg}$ & $31.50 \mathrm{fg}$ & $31.83 \mathrm{~cd}$ \\
\hline Organic manure (O.M.) & $27.31 \mathrm{fg}$ & $29.19 \mathrm{def}$ & $28.25 \mathrm{~d}$ & $28.80 \mathrm{hi}$ & $30.82 \mathrm{gh}$ & $29.81 \mathrm{~d}$ \\
\hline G.M. + Nitro. & $39.46 \mathrm{a}$ & $33.37 \mathrm{~b}$ & $36.42 \mathrm{a}$ & $38.65 \mathrm{a}$ & $35.16 \mathrm{~cd}$ & $36.91 \mathrm{a}$ \\
\hline G.M. + O.M. & $32.94 \mathrm{bc}$ & $32.12 \mathrm{bc}$ & $32.53 \mathrm{bc}$ & $33.56 \mathrm{def}$ & 34.60 cde & $34.08 \mathrm{abc}$ \\
\hline Nitro. + O.M. & $28.42 \mathrm{ef}$ & $33.94 \mathrm{~b}$ & $31.18 \mathrm{c}$ & $30.18 \mathrm{gh}$ & 35.62 cde & $32.90 \mathrm{bc}$ \\
\hline G.M. + Nitro. + O.M. & $31.94 \mathrm{bc}$ & $33.78 \mathrm{~b}$ & $32.86 \mathrm{bc}$ & $32.87 \mathrm{efg}$ & $36.28 \mathrm{bc}$ & $34.58 \mathrm{abc}$ \\
\hline \multicolumn{7}{|c|}{ Specific effect } \\
\hline NPK (control) & $24.98 \mathrm{c}$ & $25.67 \mathrm{~b}$ & $25.33 \mathrm{~b}$ & $25.18 \mathrm{c}$ & $27.18 \mathrm{~b}$ & $26.18 \mathrm{~b}$ \\
\hline Mycorrhizae & $35.64 \mathrm{a}$ & $32.53 \mathrm{a}$ & $34.09 \mathrm{a}$ & $35.68 \mathrm{a}$ & $34.60 \mathrm{a}$ & $35.14 \mathrm{a}$ \\
\hline Azotobacter & $32.81 \mathrm{ab}$ & $32.86 \mathrm{a}$ & $32.84 \mathrm{a}$ & $32.18 \mathrm{~b}$ & $34.64 \mathrm{a}$ & $33.41 \mathrm{a}$ \\
\hline Organic manure & $30.15 \mathrm{~b}$ & $32.26 \mathrm{a}$ & $31.21 \mathrm{a}$ & $31.34 \mathrm{~b}$ & $34.33 \mathrm{a}$ & $32.84 \mathrm{a}$ \\
\hline
\end{tabular}

* H.F.: Half dose NPK fertilizers. *** F.F.: Full dose NPK fertilizers.

Means followed by the same letter, within each column, are not significantly different for each other at $1 \%$ level.

\section{REFERENCES}

Akath Singh and Singh R.P. (2004). Growth, productivity and nutrient status of olive trees as influenced by combined use of bio and chemical fertilizers. Hort. J., 17(2): $125-130$.

A.O.A.C. (1985). Methods of Analysis of the Association of Official Agricultral Chemist. $8^{\text {th }}$ Ed. Washington, D.C.

Ashraf M., Ali Q. and Rha E.S. (2005). The effect of applied nitrogen on the growth and nutrient concentration of Kalonji (Nigella sativa). Australian J. of Experimental Agric., 45(4): 459-463.

Das A.K., Sadhu M.K. and Sam M.G. (1991). Effect of different levels of $\mathrm{N}$ and $\mathrm{P}$ levels on growth and yield of black cumin (Nigella sativa Linn). Hort. J., 4(1): 41-47.

Datta S., Mini-Poduval Basak, J. and Chatterjee R. (2001). Fertilizer trial on cumin black (Nigella sativa Linn.) in alluvial zone of West Bengal. Environment and Ecology, 19(4): 920-922.

Duncan D.B. (1955). Multiple range and multiple F. Tests. Biometrics, 11: 1-42.

El-Deeb S., Mohamed S.M., El-Zahawy N.H. and El-Genral E.A. (1993). Effect of nitrogen sources and levels on the growth, seed yield and oil content of Nigella sativa $\mathrm{L}$. plant. J. Appl. Sci., 8(6): 307-309, Egypt.
Gendiah H.M. (1987). Studies on growth and distribution of Citrus roots. Ph.D. Thesis, Fac. Agric., Moshtohor, Zagazig Univ., Benha Branch, Egypt (213 pp).

Gupta N.S., Sadavarte K.T., Mahorkar V.K., Jadhao B.J. and Dorak S.V. (1999). Effect of graded levels of nitrogen and bioinoculants on growth and yield of marigold (Tagetes erecta). J. Soils and Crops, 9(1): 80-83.

Khalid K.A., Abou-Hussien S.D. and Salman S.R. (2005). Influence of sulphur and biofertilizer (Sulphur-Oixdizing Bacteria) on the growth, oil and chemical composition of celery plant. Annals Agric. Sci. (Cairo), 50(1): 249-262.

Menge J.A., Lembright H. and Johnson E.L. (1977). Utilization of mycorrhizal fungi in citrus mummeries. Proc. Int. Citriculture, 1: 129-132.

Mousa G.T., El-Sallami I.H. and Ali E.F. (2001). Response of Nigella sativa L. to foliar application of gibberellic acid, benzyladenine, iron and zinc. Assiut $\mathrm{J}$. Agric. Sci., 32(2): 141-156.

Mukhopadhyay D. and Sem S.P. (1997). Augmentation of growth variables and yield components of plants yielding species by foliar application of diazotrophic bacteria. Dept. of Botany, 
Univ. of Kalyani, 741235, West Bengal, India. Indian J. Agric. Res., 31(1): 1-9.

Nagarajan P., Radha N.V., Kandasamy D., Oblisami G. and Jayaraj S. (1989). Effect of combined inoculation of Azopirillum brasilense and Glomus fasciculatum on mulberry. Madras Agric. J., 76(11): 601605.

Nataraja A., Farooqi A.A., Sreeramu B.S. and Srinivasappa K.N. (2003). Influence of nitrogen, phosphorus and potassium on growth and yield of black cumin (Nigella sativa L.). J. Spices and Aromatic Crops, 12(1): 51-54.

Rao A.V. and Dass H.C. (1989). Growth of fruit plants as influenced by nitrogen fixing bacteria. Annals of Arid Zone, 28(1-2): 143-147.

Ray R., Patra S.K., Ghosh K.K. and Sahoo S.K. (2005). Integrated nutrient management in Okra (Abelmoschus esculentus L.)in a river basin. Indian J. Hort., 62(3): 260-264.

Salama A.S.M. (2002). Response of some fruit species transplants and trees to organic fertilization. Ph.D. Thesis, Fac. Agric.
Moshtohor, Zagazig Univ., Benha Branch, Egypt.

Saraf R.K. and Tiwari J.P. (2004). Influence of integrated nutrient management on growth and yield of main and ratoon crop of muskolana (Abelmoschus moschatus). J. Medicinal and Aromatic Plant Sci., 26(1): 24-27.

Singh S.K., Sardar S. and Singh S. (1999). Response of nigella (Nigella sativa L.) to nitrogen and phosphorus. Vegetable Research Station, Kalyanpur, Kanpur. 280024, India Crop. Research. Hisar., 18(3): 478-479.

Srivastava A.K., Shyam S., Marathe R.A. and Singh S. (2002). Organic citrus soil fertility and plant nutrition. J. Sustainable Agric., 19(3): 5-59.

Steel R.G.D. and Torrie J.H. (1960). Principles and procedures of statistics. Mc. Graw Hill Book Company, Inc. U.S.A., pp. 448-449.

Wange S.S. and Patil P.L. (1994). Response of tuberose to biofertilzers and nitrogen. J. Maharashtra Agricultural Univ., 19(3): 484-485.

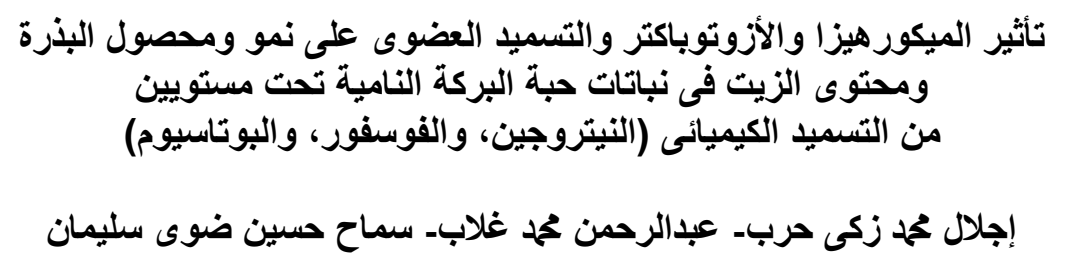

قسم النبات الزر اعى - فرع فسيولوجى النباتـ كلية الزر اعة - جامعة القاهرة

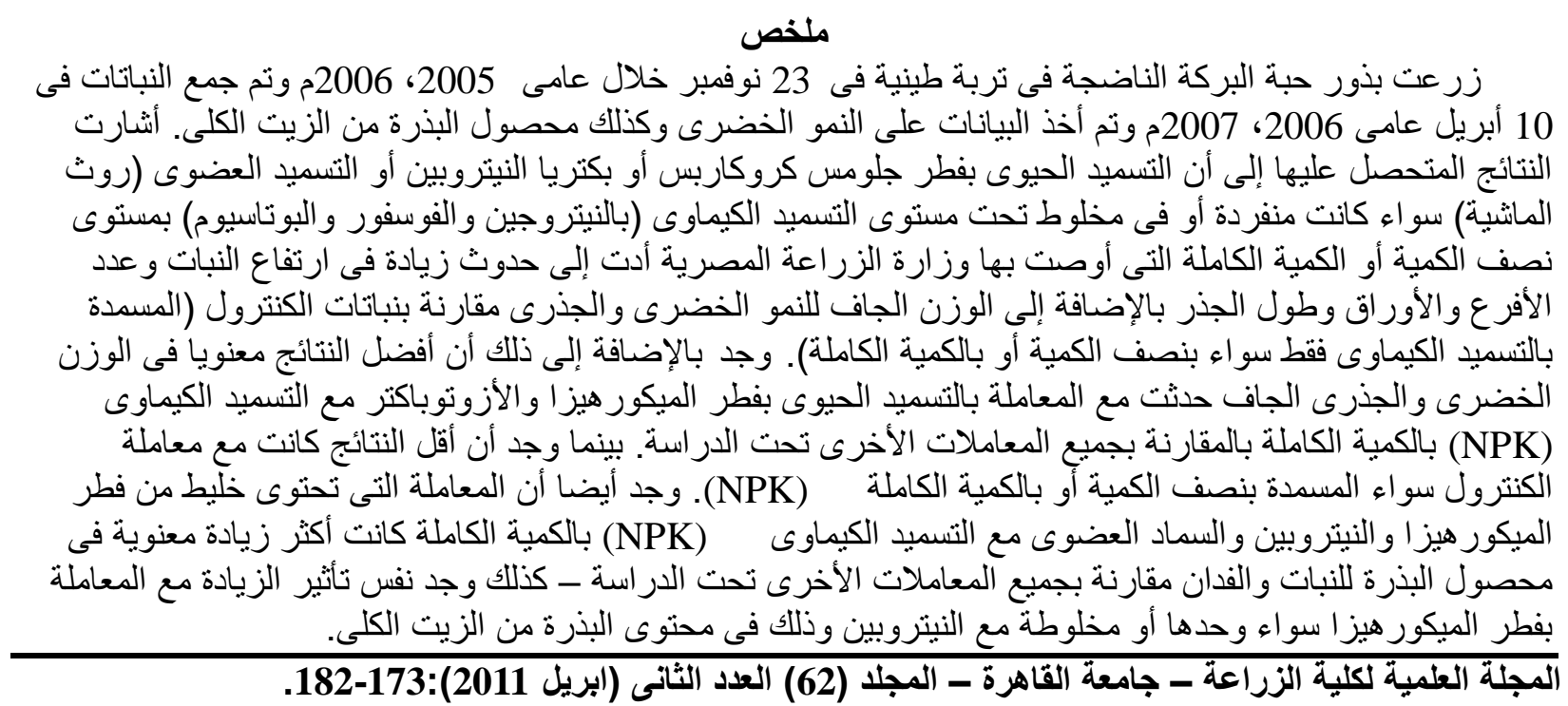

